\title{
Análise da temperatura ótima de corte para o par: metal duro revestido x aço ABNT 1045
}

\section{Analysis of the optimum cutting temperature for the pair: coated carbide $\times$ ABNT 1045 steel}

\author{
${ }^{1}$ Universidade Federal de Minas Gerais, Av. Presidente Antônio Carlos 6627, CEP: 31270-901, Belo Horizonte, Minas \\ Gerais, Brasil. \\ e-mail: vernerpereira@ufmg.br, marcelocamara@demec.ufmg.br
}

\section{RESUMO}

A otimização dos parâmetros de corte na usinagem de metais tem sido uma grande preocupação na manufatura de peças de diversos setores. Reduzir o desgaste da ferramenta e melhorar a qualidade da superfície usinada são algumas das metas do processo de corte. Nesse sentido, a temperatura de corte tem se mostrado o parâmetro mais adequado para correlacionar o desgaste da ferramenta, integridade da superfície e também força de corte. Desse modo, o objetivo geral desta pesquisa foi validar a existência de uma temperatura ótima de corte onde a mínima taxa de desgaste, mínima força de corte e mais alta qualidade da superfície usinada podiam ser alcançadas. Para tanto, foram utilizados os métodos de menor força de corte estabilizada e melhor qualidade da superfície usinada no torneamento a seco do aço ABNT 1045 recozido utilizando insertos de metal duro com revestimento PVD - (Al, Ti)N variando-se os parâmetros de corte: avanço e velocidade de corte. Em um segundo momento, para conferir se o menor desgaste coincide com a menor força de corte e melhor rugosidade da superfície usinada, foram realizados testes de desgaste da ferramenta medindo-se o desgaste de flanco, de entalhe e perda de massa dos insertos em diferentes velocidades de corte. Os resultados mostraram que, não foi possível validar a existência de uma temperatura ótima de corte pelos métodos propostos, pois foram encontradas diferentes temperaturas correspondentes ao início da estabilização das forças de corte nos avanços estudados. Os valores da rugosidade, parâmetro Ra, tiveram variações pequenas e aleatórias em um mesmo avanço com o aumento da temperatura e velocidade de corte. Os resultados dos três testes de desgaste da ferramenta apontaram para um desgaste crescente com o aumento da velocidade de corte, com isso, constatou que o menor desgaste não ocorreu na mínima força de corte.

Palavras-chave: torneamento; aço ABNT 1045; temperatura ótima; desgaste.

\begin{abstract}
The optimization of cutting parameters in metal machining has been a considerable concern in the manufacture of parts from various industries. Reducing the wear of the tool and improving the quality of the machined surface are some of the goals in the optimization of the cutting process. In this sense, the cutting temperature has been shown to be the most adequate parameter to correlate tool wear, surface integrity and also cutting force. Thus, the overall objective of this research was to validate the existence of an optimum cutting temperature where the minimum wear rate, minimum cutting force and higher quality machined surface could be achieved. For this, the methods of lower stabilized cutting force and better machined surface quality were used in the dry turning of ABNT 1045 steel annealed using coated cemented carbide inserts PVD - (Al, Ti)N varying the cutting parameters: feed rate and cutting speed. In a second moment, to check if the lesser wear coincides with the lower cutting force and better roughness of the machined surface, tool wear tests were performed measuring flank wear, notch wear and mass loss of the inserts at different cutting speeds. The results showed that it was not possible to validate the existence of an optimum cutting temperature by the proposed methods, since different temperatures corresponding to the beginning of the stabilization of the forces in the studied feeds were found and the roughness values, Ra parameter, had small and random variations in the same feed rate with increasing temperature and cutting speed. The results of the three tests of tool
\end{abstract}


wear pointed to an increasing wear with the increase of the cutting speed, with that, found that the less wear did not occur in the minimum cutting force.

Keywords: turning; ABNT 1045 steel; optimum temperature; wear.

\section{INTRODUÇÃo}

Para resumir a importância econômica, o custo de usinagem representa mais de $15 \%$ do valor de todos os produtos manufaturados nos países industrializados [1]. De acordo com DAVOODI e TAZEHKANDI [2] os parâmetros de corte na usinagem de metais afetam diretamente a eficiência, a qualidade e o custo do processamento do produto e, portanto, a otimização dos parâmetros de corte tem sido uma grande preocupação.

Ao otimizar os parâmetros do processo de usinagem, a seleção dos parâmetros do processo de usinagem é uma parte muito importante para que as operações da máquina sejam bem-sucedidas [3]. No entanto, os meios e métodos para otimizar os parâmetros de corte podem não ser efetivos na prática devido à falta de entendimento da lei e do mecanismo de usinagem [4]. Ainda de acordo com SHENG [4] os parâmetros de corte estão intimamente relacionados às ferramentas (geometria e propriedades do material), equipamentos de usinagem e materiais da peça. Portanto, é muito difícil estabelecer um modelo de otimização em conformidade com as condições reais de usinagem. Como coordenar o controle do desgaste da ferramenta com outras metas como qualidade da superfície usinada, precisão da peça usinada e tempo de produção é uma questão fundamental na otimização do processo de corte [5].

Pesquisas mostram que a vida útil da ferramenta se correlaciona bem com a relação entre as características mecânicas da ferramenta e peça apenas nas temperaturas de corte [6]. Nesse sentido, Makarow apud ASTAKHOV [7] concluiu que a temperatura de corte na interface cavaco-ferramenta é o parâmetro mais adequado para correlacionar as condições tribológicas nessa interface com o desgaste da ferramenta. Segundo Makarow para uma combinação dada de ferramenta e material a ser usinado, existe uma temperatura ótima de corte, a qual a combinação da mínima taxa de desgaste, mínima força de corte estabilizada e a mais alta qualidade da superfície usinada são alcançadas, estas relações foram denominadas por ASTAKHOV [7] como "A Primeira Lei do Corte de Metal" ou "Lei de Makarow".

Essa temperatura ótima de corte depende somente dos materiais da ferramenta e da peça, uma vez determinada, esta temperatura poderá ser usada para alcançar o ponto ótimo para vários processos de usinagem onde a mesma combinação de ferramenta/peça é usada [7]. Assim, esse modelo pode fornecer um bom controle do desgaste da ferramenta, uma vez que depende apenas do monitoramento da temperatura de corte para obter os parâmetros ótimos de corte, além de ser de fácil implantação nas indústrias.

Para estudar a relação interna entre os parâmetros ótimos de corte e a temperatura de corte, SHENG [4] realizou testes de torneamento a seco em um aço inoxidável endurecido por precipitação, dureza $38 \mathrm{HRC}$, em várias velocidades de corte, com a profundidade de corte fixada em $\mathrm{a}_{\mathrm{p}}=0,4 \mathrm{~mm}$. Pôde-se constatar que a temperatura ideal de corte, onde o desgaste da ferramenta teve o menor valor, foi bem próxima para os diferentes avanços. Os valores dos desgastes relativos foram obtidos dividindo o desgaste de flanco medido em microscópio pela área superficial usinada, utilizou-se o método termopar ferramenta-peça para as medições das temperaturas.

De forma complementar, SHENG et al. [5] com o objetivo de encontrar uma equação que relacione os parâmetros de corte na operação de fresamento de um aço inoxidável endurecido com insertos de metal duro, foi verificada uma temperatura ideal de corte, onde o desgaste da ferramenta teve o menor valor. As temperaturas na zona de corte foram obtidas por meio de uma fórmula empírica em conjunto com medições por radiação infravermelha. Os resultados indicaram que, enquanto o desgaste mínimo da ferramenta ocorre, a temperatura da ferramenta na zona de corte permaneceu a mesma, obtendo o grau mínimo de endurecimento por encruamento da superfície da peça usinada, que foi definido como a temperatura de corte ideal.

Hao et al. [8] estudaram as características de desgaste da ferramenta e o controle do desgaste pelo método baseado na temperatura ótima de corte no torneamento do Inconel 718, empregando insertos de metal duro com revestimento (TiAlN) por PVD. As temperaturas médias de corte foram medidas pelo método termopar ferramenta-peça. Os resultados mostram que a característica de desgaste da ferramenta mudou com as velocidades de corte, houve uma velocidade de corte ótima relacionada com a temperatura ótima de corte na qual os óxidos formados serviram como camada de lubrificação, nessa temperatura o desgaste da ferramenta atingiu o valor mínimo, fornecendo um bom método para o controle do desgaste da ferramenta.

Anukhin et al. [6] desenvolveram um método de seleção de condições ótimas de corte para o torneamento de ligas intermetálicas utilizadas em altas temperaturas. A temperatura ótima de corte foi determinada por meio das curvas (dureza x temperatura) dos materiais da ferramenta e peça. A temperatura que corres- 
ponde à maior diferença entre as durezas (ferramenta e peça) foi considerada a temperatura ótima de corte. Uma equação foi então elaborada para estimar esta temperatura. Por meio de testes de torneamento e medição da temperatura por radiação infravermelha, os menores desgastes da ferramenta ocorreram nas temperaturas próximas às temperaturas calculadas pela equação criada.

A temperatura ótima de corte pode ser determinada experimentalmente utilizando o teste de vida da ferramenta, onde a taxa de desgaste da ferramenta é medida em função da temperatura de corte. Contudo, o teste completo de vida da ferramenta é caro e consome muito tempo [7]. Conduzindo um número de testes com materiais de difícil usinagem, foi mostrado por [7] que a temperatura ótima de corte pode ser determinada a partir da mínima força de corte estabilizada, de um gráfico (força de corte x velocidade de corte) com diferentes avanços e profundidade de corte fixada. O início da estabilização da força de corte em um mesmo avanço corresponde a um ponto de ótimo de corte (velocidade ótima de corte e temperatura ótima de corte). Os valores das velocidades ótimas de corte $\left(\mathrm{V}_{\text {copt }}\right)$ variam em função do avanço, já a temperatura nestes pontos foi a mesma, considerada, portanto, como a temperatura ótima de corte.

Para explicar o fenômeno metalúrgico envolvido na temperatura ótima de corte $\left(\theta_{\text {opt }}\right)$ foram encontradas três causas possíveis, todas elas apontam para uma queda da ductilidade do material da peça na faixa de temperatura correspondente a $\theta_{o p t}$. Uma das causas foi proposta por [7] que evidencia o aparecimento de vazios nos contornos de grão, provocadas pelo empilhamento de discordâncias nessa região. As outras causas, encontradas em respectivamente $[9,10]$ apontam para precipitações de $\mathrm{MnS}$ e casos mais específicos, a ocorrência de óxidos de alumínio complexos, provocando a fragilização do aço.

Diante do cenário exposto, o objetivo geral desta pesquisa foi validar a existência da temperatura ótima de corte no torneamento a seco do aço ABNT 1045 recozido utilizando insertos de metal duro com revestimento PVD - (Al, Ti)N. Os objetivos específicos foram encontrar um gama de velocidades ótimas de corte para os avanços recomendados pela fabricante da ferramenta e avaliar a influência do aumento da temperatura nas forças de usinagem, rugosidade Ra e desgaste da ferramenta.

\section{MATERIAIS E MÉTODOS}

Os corpos de prova, na forma de barras cilíndricas, utilizados neste estudo foram confeccionados em aço ABNT 1045 laminado a quente com diâmetro inicial de $76 \mathrm{~mm}$ e comprimento de $300 \mathrm{~mm}$. A fim de homogeneizá-los, os corpos de prova foram recozidos a uma temperatura de $840{ }^{\circ} \mathrm{C}$ por $2 \mathrm{~h} 45 \mathrm{~min}$ e resfriados ao forno até atingir a temperatura ambiente, indicando uma dureza final de $85 \mathrm{HRB}$ e resistência a tração de 636 MPa. A composição química deste aço obtida por espectrometria de emissão ótica pode ser vista na Tabela 1.

Tabela 1: Composição química dos corpos de prova.

\begin{tabular}{l|c|c|c|c|c|c|c|c|c|c}
\hline ELEMENTO QUímICO & $\mathrm{C}$ & $\mathrm{Si}$ & $\mathrm{Mn}$ & $\mathrm{P}$ & $\mathrm{S}$ & $\mathrm{Cr}$ & $\mathrm{Cu}$ & $\mathrm{Mo}$ & $\mathrm{Ni}$ & $\mathrm{Al}$ \\
\hline COMPOSIÇÃO (\%) & 0,48 & 0,15 & 0,70 & 0,03 & 0,02 & 0,10 & 0,29 & 0,02 & 0,07 & 0,01 \\
\hline
\end{tabular}

Os ensaios de usinagem foram realizados em um torno CNC ROMI modelo Centur 30 s $(5,5 \mathrm{~kW}$ de potência e rotação máxima de 3500 rpm, com comando numérico Mach 9).

Foram utilizados insertos de tornear de metal duro revestido da empresa Mitsubishi Materials com geometria CNMG120408, classificação ISO P20 a P30, quebra-cavaco MA, revestimento VP15TF - (Al, Ti)N pela técnica PVD e suporte com geometria PCLNR 2020K-12. Este inserto é empregado na usinagem média a desbaste de aço carbono e aço liga com os parâmetros de corte recomendados: $V_{c}=90$ a $160 \mathrm{~m} / \mathrm{min}, \mathrm{f}=0,2$ a $0,5 \mathrm{~mm} / \mathrm{rev}$ e $\mathrm{a}_{\mathrm{p}}=0,3$ a $4,0 \mathrm{~mm}$ [11].

Na primeira etapa, para validar a existência da temperatura ótima de corte foi utilizado o método de menor força de corte estabilizada proposto por [7] e melhor qualidade da superfície usinada variando-se a velocidade de corte $\left(\mathrm{V}_{\mathrm{c}}\right)$ e avanço $(\mathrm{f})$. Em cada ensaio será medido a temperatura de corte, força de usinagem e rugosidade superficial $\mathrm{Ra}$.

Em uma segunda etapa, para verificar se realmente o menor desgaste ocorre coincidentemente na menor força de corte e na melhor qualidade da superfície usinada foram realizados medições de desgaste nos insertos em diferentes velocidades de corte. 


\subsection{Ensaios da temperatura, força e rugosidade no torneamento (etapa 1)}

$\mathrm{Na}$ Etapa 1 foram realizados os ensaios de temperatura, força e rugosidade no torneamento, totalizando setenta e dois (72) ensaios, sendo uma réplica para cada, Em cada ensaio foi medido em uma mesma operação de torneamento a temperatura e força de corte, ao término da operação o corpo de prova era retirado do torno e realizavam-se as medições de rugosidade em uma bancada.

Foi observado por meio de testes que cinco segundos (5s) de corte eram suficientes para a estabilização da força e temperatura na operação de torneamento. Por segurança foi definido então um tempo de corte $\left(t_{c}\right)$ de 10s. Para manter constante este tempo em cada ensaio, foi utilizada a Equação 1, onde o valor do percurso de avanço $\left(\mathrm{L}_{\mathrm{f}}\right)$ era calculado em função dos parâmetros de corte e diâmetro momentâneo (D) do corpo de prova. Com este valor calculado ajustava-se o programa no torno CNC em cada ensaio.

$$
L_{f}[\mathrm{~mm}]=\frac{V_{c}[\mathrm{~m} / \mathrm{min}] \cdot 1000 \cdot f[\mathrm{~mm} / \mathrm{rev}] \cdot t_{c}[\mathrm{~s}]}{\pi \cdot D[\mathrm{~mm}] \cdot 60}
$$

Para amenizar a influência do desgaste do inserto nos resultados, foi definido que a cada quatro ensaios, ou seja, aproximadamente 40s de tempo de corte, a aresta do inserto era trocada. Além disso, para diminuir a flexão do corpo de prova durante a usinagem e possíveis vibrações o diâmetro menor torneado foi de $40 \mathrm{~mm}$.

Para a coleta de dados da temperatura, utilizou-se um pirômetro infravermelho Raytek modelo $M M 2 M L$ com faixa de operação de $300^{\circ} \mathrm{C}$ a $1100^{\circ} \mathrm{C}$ e tempo de resposta de $2 \mathrm{~ms}$. O software utilizado para aquisição do sinal de temperatura foi o Multidrop Rev.5.3.1 criado pela Data Temp. O ponto focal foi direcionado sobre a ponta do inserto na superfície de saída (direção circunferencial), o diâmetro do ponto focal foi de aproximadamente $1 \mathrm{~mm}$. Foi adotada neste trabalho a emissividade de 0,77 em todos os ensaios de temperatura, essa emissividade foi obtida na temperatura de $570^{\circ} \mathrm{C}$ de um cavaco do aço AISI 1045 , segundo a pesquisa de HEIGEL e WHITENTON [12].

Para medição das forças de usinagem durante o torneamento, foi utilizado o dinamômetro Kistler modelo 9272 conectado ao amplificador multicanal também da Kistler modelo 5073A. Através do software ManuWare da Kistler, instalado em um computador e conectado ao amplificador, foram ajustados os limites máximos das forças e definidos os fatores de conversão de volts para newtons. Para a aquisição de dados utilizou-se a placa da National Instruments - modelo USB-6366 conectada ao amplificador e ao computador, o software utilizado para aquisição do sinal de força em volts foi o NI LabView SignalExpress 2013 e posteriormente os dados coletados foram exportados para o Microsoft Excel 2007, a taxa de aquisição de dados foi de $240 \mathrm{~Hz}$. Um modelo esquemático desse arranjo pode ser visto na Figura 1. Durante o torneamento foi realizada a aquisição dos sinais de força de usinagem nas três direções básicas que agem diretamente na cunha cortante, são elas: $F_{c}$ - força de corte, $F_{f}$ - força de avanço e $F_{p}$ - força passiva.

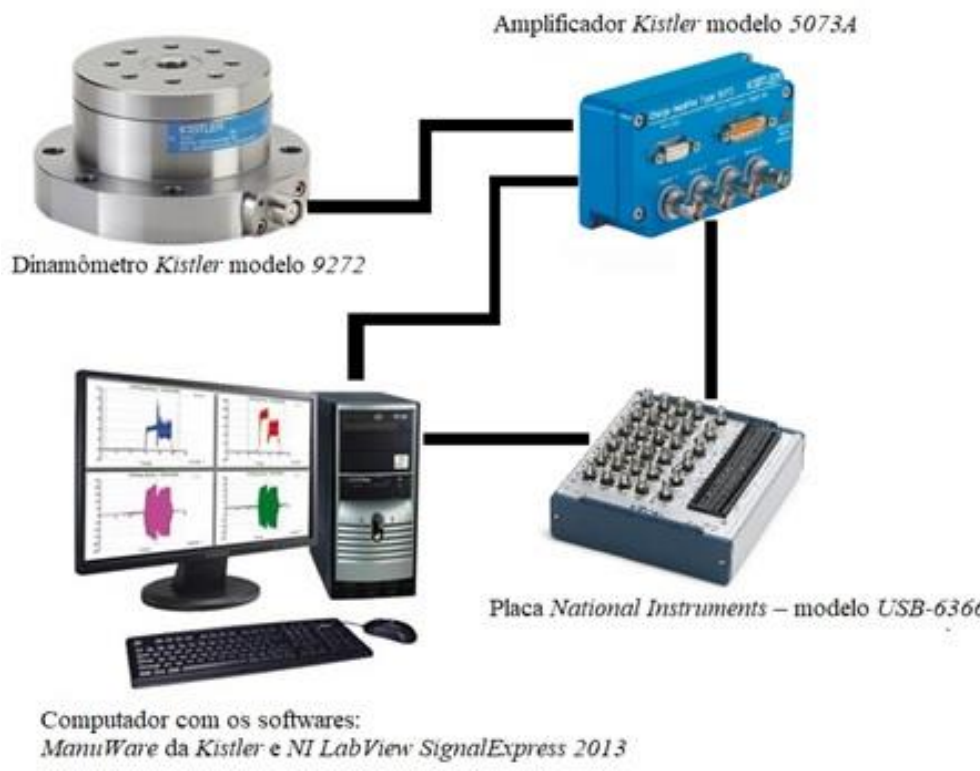

Figura 1: Modelo esquemático da obtenção das forças de usinagem - Etapa 1. Fonte: Adaptado de OLIVEIRA [13]. 
A qualidade da superfície usinada foi avaliada por meio da rugosidade, que são desvios microgeométricos originados pelo próprio processo de formação do cavaco, vibrações da ferramenta, aresta postiça de corte, marcas de avanço da ferramenta com a peça, etc. O parâmetro de rugosidade superficial Ra (desvio aritmético médio) foi adotado por ser amplamente empregado como parâmetro de controle de processo e como representa um valor médio, é um parâmetro estável, que não recebe influência de efeitos ocasionais. As medições da rugosidade foram coletadas com um rugosímetro portátil Taylor Hobson, modelo Precision Surtronic 25, realizando a medição em cada superfície usinada em três posições diferentes, afastadas $120^{\circ}$ umas das outras e o valor médio foi calculado. Foi empregado o comprimento de amostragem de 2,5 $\mathrm{mm}$ com o comprimento de avaliação de $12,5 \mathrm{~mm}$ (recomendados para os valores de Ra entre 2 e $10 \mu \mathrm{m}$ ) e o comprimento de amostragem de $0,8 \mathrm{~mm}$ com o comprimento de avaliação de $4 \mathrm{~mm}$ (para os valores de Ra entre 0,1 e $2 \mu \mathrm{m}$ ).

\subsection{Ensaio de desgaste da ferramenta (etapa 2)}

Para realização dos ensaios de desgaste foram utilizados quatro corpos de prova de (Ø 76 x 300$) \mathrm{mm}$, foram escolhidos quatro velocidades de corte $\left(\mathrm{V}_{\mathrm{c}}\right)$ e um avanço (f). Para poder comparar o desgaste obtido em cada $\mathrm{V}_{\mathrm{c}}$, foi definido o mesmo comprimento total de contato (L), consequentemente o mesmo volume usinado. $\mathrm{O}$ percurso de avanço $\left(\mathrm{L}_{\mathrm{f}}\right)$ foi de $280 \mathrm{~mm}$, o diâmetro inicial dos corpos de prova foi de $74 \mathrm{~mm}$ e diâmetro final de $30 \mathrm{~mm}$. A partir destes dados, foram calculados por meio da Equação 2 o comprimento total de contato (L) de cada teste, onde (r) é o raio momentâneo da barra em [mm], também foi calculado utilizando a Equação 3 o tempo de corte $\left(\mathrm{t}_{\mathrm{c}}\right.$ ). Para cada teste, ou seja, para cada $\mathrm{V}_{\mathrm{c}}$ foi utilizado um inserto e apenas uma aresta de corte.

$$
\begin{aligned}
& L[m]=\sum_{r=15}^{37} \frac{2 \pi \cdot r \cdot L_{f}}{f \cdot 1000} \\
& t_{c}[\mathrm{~min}]=\sum_{r=15}^{37} \frac{2 \pi \cdot r \cdot L_{f}}{f \cdot 1000 \cdot V_{c}}
\end{aligned}
$$

A medição do desgaste se deu de três maneiras, por meio da perda de massa dos insertos, da medição do desgaste de flanco e desgaste de entalhe. Devido às sujeiras e material aderido presentes na superfície dos insertos após a operação de torneamento, foi necessário realizar uma limpeza ultrassônica a fim de não interferir na medição do desgaste.

O equipamento utilizado para medir a perda de massa dos insertos foi uma balança de precisão Shimadzu modelo AX200, carga máxima $200 \mathrm{~g}$ e resolução de $0,1 \mathrm{mg}$, os insertos foram pesados antes dos testes e após o procedimento de limpeza.

Para as medições dos desgastes de flanco e entalhe foi utilizado um estereomicroscópio binocular Olympus modelo SZ61, ampliação máxima de 4,5 x, com uma câmera acoplada a um computador. Com as imagens obtidas por meio do estereomicroscópio, as medições dos desgastes foram feitas com o auxilio do software AxioVision Rel. 4.82012 e também das imagens de uma régua de calibração microscopia geradas na mesma ampliação dos insertos desgastados. A definição das zonas de medições, bem como os critérios dos desgastes de flanco e entalhe, foram estabelecidos conforme a norma ISO 3685 [14].

\section{RESULTADOS}

\subsection{Temperatura, força e rugosidade no torneamento (etapa 1)}

Na Figura 2 pode ser observado o gráfico da força de corte e temperatura em função de uma ampla gama de velocidades de corte, onde foi possível observar conforme previsto, certa estabilização das forças de corte nos avanços estudados. As linhas verticais pontilhadas indicam as velocidades de corte correspondentes com o início dessa estabilização.

Conforme demonstrado na Figura 2 por meio das linhas pontilhadas horizontais, foram encontradas distintas temperaturas de corte para as $\mathrm{V}_{\mathrm{c}}$ correspondentes ao início da estabilização das forças. Verifica-se que essas temperaturas aumentaram com o avanço e para os pares de avanços $(0,2$ e 0,3$) \mathrm{mm} / \mathrm{rev}$ e $(0,4$ e 0,5$)$ $\mathrm{mm} / \mathrm{rev}$ essas temperaturas foram próximas, ver Tabela 2.

Do mesmo modo como retratado anteriormente para a força de corte, os resultados das temperaturas, força de avanço e passiva em função da velocidade de corte, foram observadas também distintas temperaturas de corte para as $V_{c}$ correspondentes ao início da estabilização dessas forças, com exceção das forças $F_{f}$ $(f=0,2)$ e $F_{p}(f=0,2)$, onde a forças tiveram apenas pequenas variações. Constatou-se que as $V_{c}$ corresponden- 
tes ao início da estabilização das forças, ou seja, possíveis velocidades ótimas de corte $\left(\mathrm{V}_{\text {copt }}\right)$ foram próximas para as três componentes da força de usinagem nos avanços estudados, ver Tabela 3. Além disso, essas possíveis $\left(\mathrm{V}_{\text {copt }}\right)$ tiveram os valores acima das velocidades de corte recomendadas pelo fabricante dos insertos de 90 a $160 \mathrm{~m} / \mathrm{min}$.

Tabela 2: $\mathrm{V}_{\mathrm{c}}$ e Temp. associados ao início da estabilização das $\mathrm{F}_{\mathrm{c}}$.

\begin{tabular}{c|c|c}
\hline $\begin{array}{c}\text { Avanço } \\
{[\mathrm{mm} / \mathbf{r e v}]}\end{array}$ & $\begin{array}{c}\mathbf{V}_{\mathbf{c}}[\mathbf{m} / \mathbf{m i n}] \mathbf{d a} \\
\mathbf{F}_{\mathbf{c}} \text { estabilizada }\end{array}$ & $\begin{array}{c}\text { Temp. }\left[{ }^{\circ} \mathbf{C}\right] \text { da } \\
\mathbf{F}_{\mathrm{c}} \text { estabilizada }\end{array}$ \\
\hline $\mathbf{0 , 5}$ & 240 & 460 \\
\hline $\mathbf{0 , 4}$ & 250 & 442 \\
\hline $\mathbf{0 , 3}$ & 190 & 410 \\
\hline $\mathbf{0 , 2}$ & 200 & 402 \\
\hline
\end{tabular}

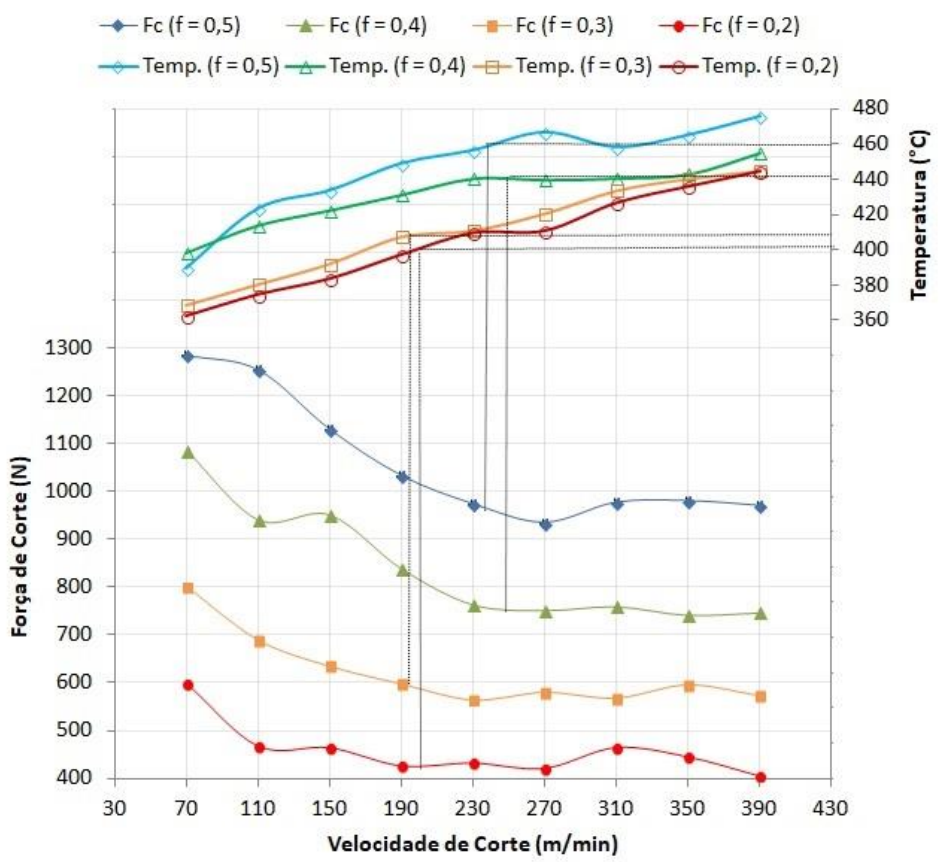

Figura 2: Relação da velocidade de corte com a temperatura e força de corte $\left(\mathrm{F}_{\mathrm{c}}\right)$ nos avanços: $\mathrm{f}=(0,2,0,3,0,4$ e 0,5$)$ $\mathrm{mm} / \mathrm{rev}$ e $\mathrm{a}_{\mathrm{p}}=1 \mathrm{~mm}$.

Tabela 3: Comparação entre as possíveis $\mathrm{V}_{\text {copt }}$ das forças de usinagem estabilizadas.

\begin{tabular}{c|c|c|c}
\hline $\begin{array}{c}\text { Avanço } \\
{[\mathbf{m m} / \mathbf{r e v}]}\end{array}$ & $\begin{array}{c}\mathbf{V}_{\text {copt }}\left(\mathbf{F}_{\mathrm{c}}\right) \\
{[\mathbf{m} / \mathbf{m i n}]}\end{array}$ & $\begin{array}{c}\mathbf{V}_{\text {copt }}\left(\mathbf{F}_{\mathrm{f}}\right) \\
{[\mathbf{m} / \mathbf{m i n}]}\end{array}$ & $\begin{array}{c}\mathbf{V}_{\text {copt }}\left(\mathbf{F}_{\mathbf{p}}\right) \\
{[\mathbf{m} / \mathbf{m i n}]}\end{array}$ \\
\hline $\mathbf{0 , 5}$ & 240 & 270 & 270 \\
\hline $\mathbf{0 , 4}$ & 250 & 270 & 270 \\
\hline $\mathbf{0 , 3}$ & 190 & 190 & 190 \\
\hline $\mathbf{0 , 2}$ & 200 & - & - \\
\hline
\end{tabular}

Foi encontrado um desvio padrão médio de $0,6 \%$ em relação às temperaturas médias, o maior desvio padrão foi de $2,9 \%$. Segundo RAYTEC [15], manual do fabricante do pirômetro infravermelho utilizado nos ensaios, o instrumento apresenta uma precisão de $\pm\left(0,3 \%\right.$ da leitura $\left.+2^{\circ} \mathrm{C}\right)$ no intervalo de medição de $(300$ a 1100$)^{\circ} \mathrm{C}$. Portanto, a incerteza do instrumento pode ter sido significativa na dispersão encontrada, pois apresentou valores próximos.

Os desvios padrão das medições das forças de usinagem também foram calculados, obtendo um desvio 
padrão médio $\left(\sigma_{\text {méd. }}\right.$ ) de 3,3\% e maior desvio padrão $\left(\sigma_{\text {maior }}\right) 9,3 \%$ para as forças de corte. As forças de avanço e passiva apresentaram resultados similares, $\sigma_{\text {méd. }}=2,2 \%$ e $2,4 \%, \sigma_{\text {maior }}=12,2 \%$ e $11,2 \%$, respectivamente. De acordo com os manuais do dinamômetro KISTLER [16] e amplificador KISTLER [17], os instrumentos apresentam uma precisão de $2 \%$ e $0,5 \%$, respectivamente, estes valores não inclui erros devido a fontes externas, adaptações mecânicas dos sensores e influências do meio ambiente. Assim como na medição da temperatura, a precisão dos instrumentos e desvio padrão médio tiveram valores próximos.

A Figura 3 apresenta o gráfico da rugosidade (Ra) e temperatura em função das velocidades de corte. Com exceção da curva $\mathrm{Ra}(\mathrm{f}=0,5)$, não foi possível determinar com precisão um ponto de mínima rugosidade de modo claro. As curvas $\mathrm{Ra}(\mathrm{f}=0,4)$ e $\mathrm{Ra}(\mathrm{f}=0,2)$ tiveram variações pequenas com o aumento da temperatura e velocidade de corte, já a curva $\mathrm{Ra}(\mathrm{f}=0,3)$ apresentou dois mínimos em $\mathrm{V}_{\mathrm{c}}$ bem distintas e a curva $\mathrm{Ra}$ $(\mathrm{f}=0,5)$ apresentou a mínima rugosidade na $\mathrm{V}_{\mathrm{c}}=150 \mathrm{~m} / \mathrm{min}$, divergente da possível $\left(\mathrm{V}_{\text {copt }}\right)$ encontrada com a estabilização da força de corte em $240 \mathrm{~m} / \mathrm{min}$.

Foi encontrado um desvio padrão médio de 1,9\% e maior desvio padrão de 9,8\%. O rugosímetro utilizado apresenta uma precisão de $2 \%$ da mesma forma semelhante ao desvio médio padrão.

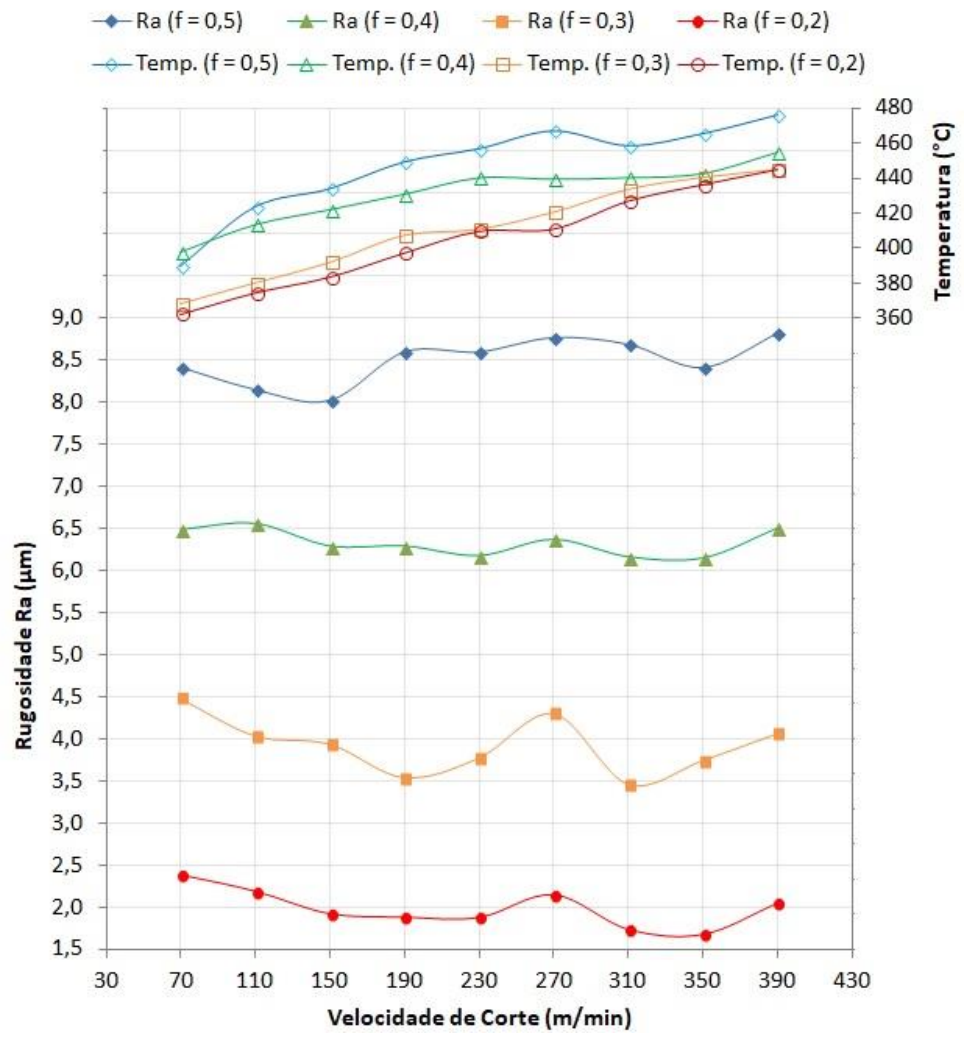

Figura 3: Relação da velocidade de corte com a temperatura e Rugosidade (Ra) nos avanços: $f=(0,2,0,3,0,4$ e 0,5$)$ $\mathrm{mm} / \mathrm{rev}$ e $\mathrm{a}_{\mathrm{p}}=1 \mathrm{~mm}$.

\subsection{Desgaste da ferramenta (etapa 2)}

Aplicando as Equações 2 e 3 foi calculado o comprimento total de contato e tempo total de contato exposto na Tabela 4. Para o teste $V_{c}=350(\mathrm{~m} / \mathrm{min})$, a aresta do inserto ficou impossibilitada de continuar o ensaio devido a um grande desgaste ocorrido em apenas $\mathrm{L}=600 \mathrm{~m} \mathrm{e}_{\mathrm{c}}=1,7 \mathrm{~min}$, nos outros testes o comprimento total de contato foi mantido fixo em aproximadamente $3900 \mathrm{~m}$.

A Figura 4 apresenta o resultado da perda de massa dos insertos em função das velocidades de corte. Devido à perda de massa ter sido bem pequena para as $\mathrm{V}_{\mathrm{c}} 70$ e 110, estas tiveram as incertezas de medição maiores que o próprio valor, sendo, portanto valores com baixa precisão, além disso, apresentaram valores estatisticamente semelhantes. Porém, o que se observa nesse gráfico é uma perda de massa crescente com o aumento da $V_{c}$, entre as $V_{c} 110$ e 230 o aumento foi entorno de 5 vezes e entre as $V_{c} 230$ e 350 o aumento foi mais de 22 vezes. As incertezas de medição foram calculadas considerando o desvio padrão, resolução limitada da balança e incerteza expandida com grau de liberdade para quatro medições. 
Tabela 4: Comprimento e tempo total de contato

\begin{tabular}{c|c|c}
\hline $\begin{array}{c}\text { Veloc. de corte } \\
{[\mathbf{m} / \mathbf{m i n}]}\end{array}$ & $\begin{array}{c}\text { Comprimento } \\
\text { total de contato }(\mathbf{L})\left[\mathbf{\times 1 0 ^ { 3 }} \mathbf{~} \mathbf{~}\right]\end{array}$ & $\begin{array}{c}\text { Tempo total } \\
\text { de contato }\left(\mathbf{t}_{\mathbf{c}}\right)[\mathbf{m i n}]\end{array}$ \\
\hline $\mathbf{V}_{\mathbf{c}} \mathbf{7 0}$ & 3,9 & 55,7 \\
\hline $\mathbf{V}_{\mathbf{c}} \mathbf{1 1 0}$ & 3,9 & 35,5 \\
\hline $\mathbf{V}_{\mathbf{c}} \mathbf{2 3 0}$ & 3,9 & 17,0 \\
\hline $\mathbf{V}_{\mathbf{c}} \mathbf{3 5 0}$ & 0,6 & 1,7 \\
\hline
\end{tabular}

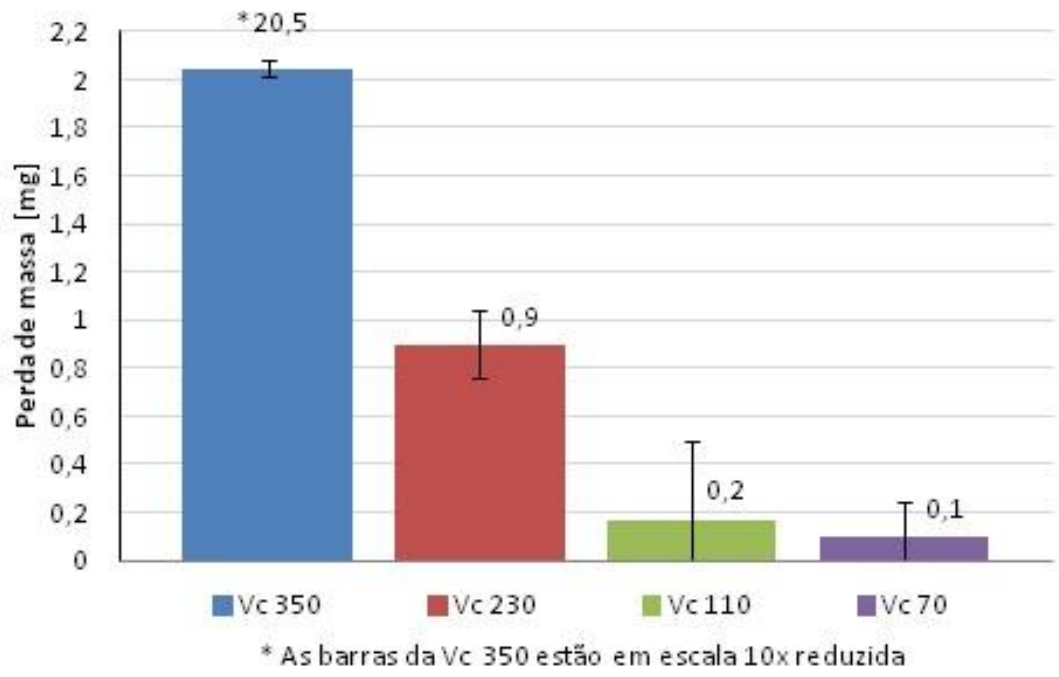

Figura 4: Perda de massa dos insertos em função das velocidades de cortes: $f=0,3 \mathrm{~mm} / \mathrm{rev}, \mathrm{ap}=1 \mathrm{~mm}, \mathrm{~L}=3900 \mathrm{~m}\left(\mathrm{~V}_{\mathrm{c}}\right.$ $70,110$ e 230$)$ e $\mathrm{L}=600 \mathrm{~m}\left(\mathrm{~V}_{\mathrm{c}} 350\right)$.

A Figura 5 e 6 apresentam os resultados dos desgastes de flanco e entalhe em função das velocidades de corte. Foi observado em ambos um desgaste crescente com o aumento da $V_{c}$, com exceção para as $V_{c} 70$ e 110 , que também apresentaram valores estatisticamente semelhantes. Os desgastes de flanco apresentaram distribuição regular ao longo da aresta principal de corte, sendo utilizado conforme recomendação da norma ISO 3685 [14], desgaste de flanco médio (VBB). O desgaste de entalhe (VBN) foi medido após a área de contato ferramenta-peça, correspondente neste ensaio com a profundidade de corte. Devido ao desgaste excessivo para $V_{c} 350$, o VBB e VBN foram coincidentes. As incertezas de medição foram calculadas a partir dos desvios padrão obtidas nas medições das imagens geradas em diferentes ampliações e incerteza expandida com grau de liberdade para quatro medições.

Durante o ensaio de desgaste foi realizado um acompanhamento da evolução do desgaste de flanco por meio da geração das imagens dos insertos a cada aproximadamente $650 \mathrm{~m}$ de comprimento total de contato (L), totalizando 6 verificações para cada $V_{c}$. A exceção foi a $V_{c} 350$, onde a aresta de corte do inserto já ficou inutilizada na primeira verificação. Após as medições de VBB nas 6 verificações constatou-se com boa aproximação uma taxa de desgaste de flanco constante. Portanto, os resultados apresentados nessa etapa para as $V_{c} 70,110$ e 230 foram obtidos com os insertos no Estágio II (taxa de desgaste constante) da curva padrão de desgaste. 


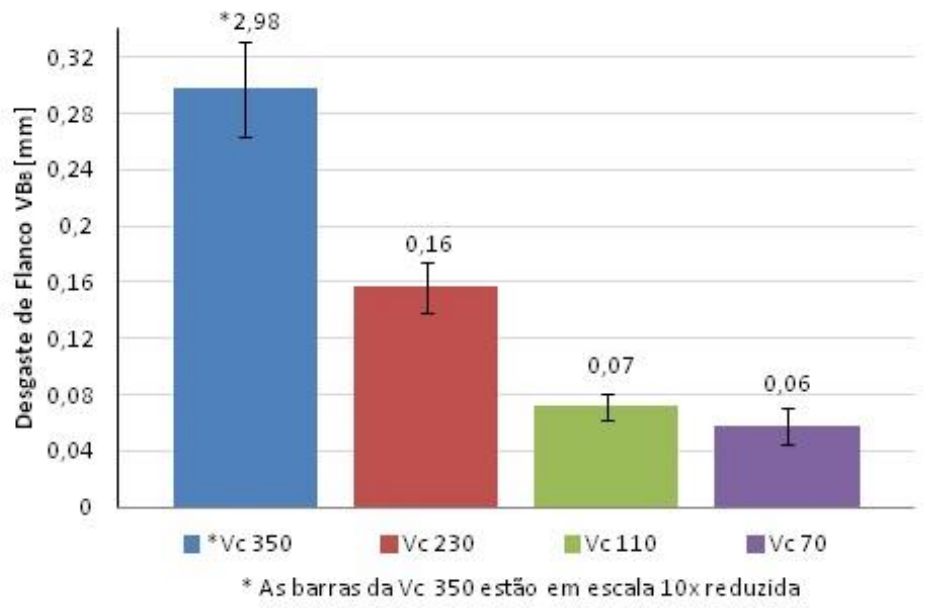

Figura 5: Desgaste de flanco médio em função das velocidades de cortes: $f=0,3 \mathrm{~mm} / \mathrm{rev}, \mathrm{ap}_{\mathrm{p}}=1 \mathrm{~mm}, \mathrm{~L}=3900 \mathrm{~m}\left(\mathrm{~V}_{\mathrm{c}} 70\right.$, 110 e 230$)$ e $\mathrm{L}=600 \mathrm{~m}$ ( $\left.\mathrm{V}_{\mathrm{c}} 350\right)$.

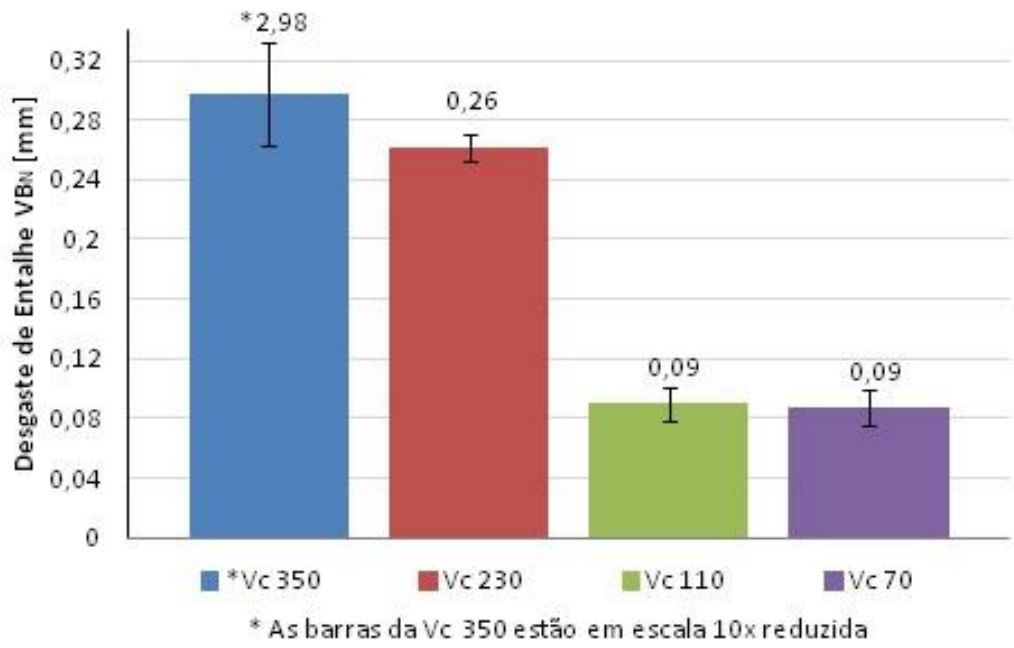

Figura 6: Desgaste de entalhe em função das velocidades de cortes: $f=0,3 \mathrm{~mm} / \mathrm{rev}, \mathrm{ap}=1 \mathrm{~mm}, \mathrm{~L}=3900 \mathrm{~m}\left(\mathrm{~V}_{\mathrm{c}} 70,110 \mathrm{e}\right.$ 230) e $\mathrm{L}=600 \mathrm{~m}\left(\mathrm{~V}_{\mathrm{c}} 350\right)$.

A Figura 7 apresenta o quadro de algumas das imagens obtidas pelo estereomicroscópio binocular utilizadas nas medições dos desgastes VBB e VBN, evidenciando a superfície de saída (A $\gamma$ ) e superfície principal de folga $(\mathrm{A} \alpha)$ para as diferentes $\mathrm{V}_{\mathrm{c}}$. As imagens têm ampliação de 2,5 x com exceção da $\mathrm{A} \alpha$ para $\mathrm{V}_{\mathrm{c}} 350$ com ampliação de 1,5 x. 


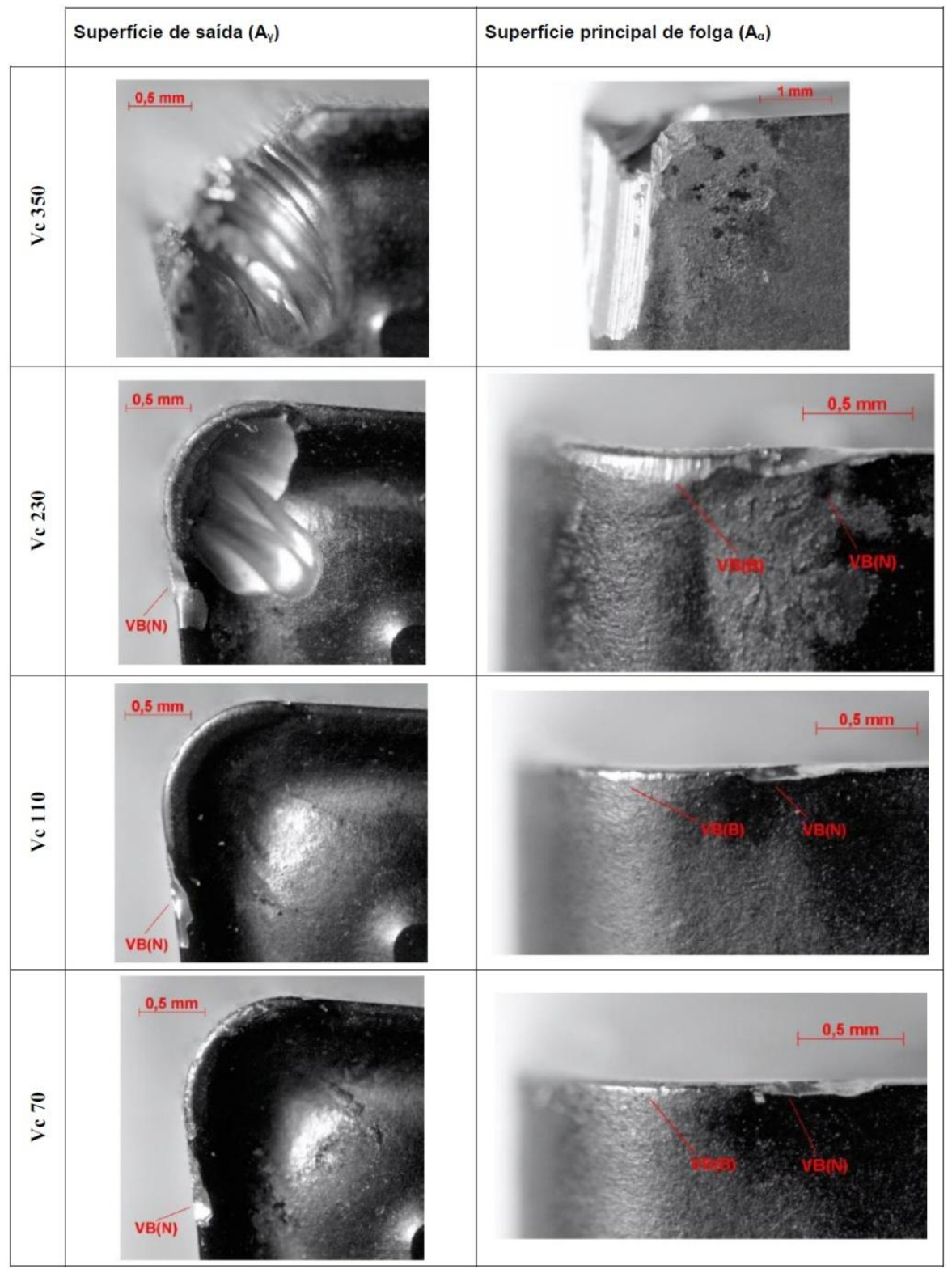

Figura 7: Imagens dos insertos desgastados: $\mathrm{f}=0,3 \mathrm{~mm} / \mathrm{rev}$ e ap $=1 \mathrm{~mm}$

\section{DISCUSSÃO}

\subsection{Temperatura, força e rugosidade no torneamento (etapa 1)}

Avaliando o efeito da velocidade de corte e avanço por meio da Figura 2, foi possível verificar que a temperatura de usinagem aumentou continuamente com a $\mathrm{V}_{c}$, apresentando uma taxa de crescimento aproximadamente constante, a temperatura de usinagem também foi maior com o aumento dos valores de avanço, porém com um crescimento pequeno, sendo que em alguns pontos, a diferença foi mínima. As forças de usinagem diminuíram com o aumento da $\mathrm{V}_{\mathrm{c}}$ até as $\mathrm{V}_{\text {copt }}$ e depois seguiram aproximadamente estabilizadas, a diminuição das forças tem como causa bastante provável a queda da dureza do material dos corpos de prova com o aumento da temperatura de usinagem, a estabilização das forças pode está relacionada com a que queda da taxa de diminuição da dureza dos corpos de prova e também com o aumento do desgaste da ferramenta provocados pelo aumento da temperatura.

Comparando os resultados das forças de corte com os valores obtidos nas pesquisas de [18, 19] observou-se boa correlação. Esta comparação pode ser feita, ambas no torneamento do aço AISI 1045 por um in- 
serto de metal duro e $\mathrm{a}_{\mathrm{p}}=1 \mathrm{~mm}$.

A temperatura ótima de corte no torneamento do aço AISI 1045 por uma ferramenta de metal duro P30 encontrada por [7] foi de $860^{\circ} \mathrm{C}$. Temperatura essa bem acima das encontradas neste trabalho, temperaturas de $(402 \text { a } 460)^{\circ} \mathrm{C}$, Tabela 2.

Porém, o objetivo das medições das temperaturas não seriam encontrar a temperatura ótima de corte e sim atestar sua existência, tendo em vista que a medição por radiação infravermelho não é recomendada para medição da temperatura média de corte na interface cavaco-ferramenta.

Para atestar a existência da temperatura ótima de corte pelo método proposto por [7], as temperaturas correspondentes as $\mathrm{V}_{\mathrm{c}}$ do início da estabilização das forças de corte deveriam ser coincidentes ou próximas, porém foram identificadas distintas temperaturas, com uma variação próxima de $58^{\circ} \mathrm{C}$, ver Tabela 2. Uma possível causa para as distintas temperaturas pode está relacionado com o método utilizado. A medição por radiação infravermelho na direção circunferencial, ou seja, medindo temperatura na superfície livre do cavaco, tem maior influência na variação da espessura do cavaco por diferentes avanços do que pelo método termopar ferramenta-peça.

Foi observado na Figura 3 uma leve queda da rugosidade entre as $V_{c}(70$ a 150$) \mathrm{m} / \mathrm{min}$ para todos os avanços, provavelmente por causa da redução da força de usinagem. Para os valores das rugosidades entre as $V_{c}(150$ a 390) $\mathrm{m} / \mathrm{min}$ acredita-se que os resultados tenham mais influência do desgaste do inserto e possíveis ranhuras da superfície usinada pelos cavacos do que com o aumento das temperaturas, isto se explica devido ao caráter aleatório nas curvas $\mathrm{Ra}(\mathrm{f}=0,5)$ e $\mathrm{Ra}(\mathrm{f}=0,3)$ e também por ter apresentado níveis médios de rugosidade ( $R a)$ estatisticamente iguais entre os valores de um mesmo avanço nas curvas $R a(f=0,4)$ e $R a(f=0,2)$.

Verificou-se também que possivelmente não houve formação da aresta postiça de corte (APC) devido às pequenas variações da rugosidade. Conforme citado nos experimentos de [20,21], a rugosidade máxima na usinagem dos aços ABNT 1050 e AISI 1045, respectivamente, ocorre na maior formação da APC, aumentando-se a velocidade de corte, a rugosidade abaixa e praticamente se estabiliza.

\subsection{Desgaste da ferramenta (etapa 2)}

A partir dos três gráficos apresentados nas Figuras 4,5 e 6 e o quadro das imagens dos insertos desgastados (Figura 7) é possível constatar que o menor desgaste da ferramenta ocorreu na $V_{c} 70$ com valores bastante próximos da $\mathrm{V}_{\mathrm{c}} 110$ principalmente no desgaste de entalhe. $\mathrm{O}$ maior desgaste ocorrido em $\mathrm{V}_{\mathrm{c}}$ maiores possivelmente está relacionado com o aumento da temperatura de usinagem, conforme citado por Machado et al. [22], o aumento da temperatura reduz o limite de escoamento das ferramentas além de acelerar os mecanismos de desgaste termicamente ativados.

Por meio da análise das imagens dos insertos desgastados foram feitas as seguintes observações:

- $O$ desgaste de cratera ocorreu apenas nas $V_{c} 230$ e 350 e apresentou aparência lisa, característica do mecanismo de desgaste por difusão e típico de $\mathrm{V}_{\mathrm{c}}$ mais altas (maiores temperaturas) conforme relatado por [22].

- $\mathrm{O}$ desgaste de flanco para as $\mathrm{V}_{\mathrm{c}} 70,110$ e 230 apresentou aspecto áspero e presença de sulcos paralelos entre si, característica dos mecanismos de desgaste por adesão e abrasão e mais típico em $\mathrm{V}_{\mathrm{c}}$ baixas.

- Não foi encontrada nenhuma ocorrência de avaria (trinca, lascamento ou quebra).

\subsection{Considerações finais}

As velocidades ótimas de corte $\left(\mathrm{V}_{\text {copt }}\right)$ encontradas, ou seja, as $\mathrm{V}_{\mathrm{c}}$ onde ocorre o menor desgaste da ferramenta, foram divergentes na comparação das Etapas 1 e 2 no avanço estudado $(0,3 \mathrm{~mm} / \mathrm{rev})$. Na Etapa 1 a $\mathrm{V}_{\text {copt }}$ foi de $(190 \mathrm{~m} / \mathrm{min})$, na Etapa 2, com a hipótese de não existir outro ponto de mínimo desgaste entre as $\mathrm{V}_{\mathrm{c}}$ estudadas, o que seria pouco provável tendo em vista a tendência dos gráficos apontarem para o menor desgaste em $\mathrm{V}_{\mathrm{c}}$ menores, a $\mathrm{V}_{\text {copt }}$ foi de $(70 \mathrm{~m} / \mathrm{min})$.

Apesar da $V_{c} 70 \mathrm{~m} / \mathrm{min}$ apresentar a maior força de usinagem nas três direções básicas $\left(F_{c}, F_{f}\right.$ e $\left.F_{p}\right)$, os ensaios da Etapa 2 revelaram para essa $\mathrm{V}_{\mathrm{c}}$ um $\mathrm{VB}_{\mathrm{B}}$ de $62,5 \%$, $\mathrm{VB}_{\mathrm{N}}$ de $65,4 \%$ e massa perdida $88,8 \%$ menor em comparação com a $V_{c} 230 \mathrm{~m} / \mathrm{min}\left(\mathrm{V}_{\mathrm{c}}\right.$ mais próxima avaliada na Etapa 2 da $\mathrm{V}_{\text {copt }}=190 \mathrm{~m} / \mathrm{min}$ encontrada na Etapa 1).

Essa tendência de alcançar o menor desgaste da ferramenta em $V_{c}$ mais baixas também foi observado no pela fabricante da ferramenta [23], com condições de usinagem similares (exceção $a_{p}=1,5 \mathrm{~mm}$ ), mesmo inserto e material da peça dos ensaios da Etapa 2. Comparando as taxas de desgaste $\left(\mathrm{VB}_{\mathrm{B}} / \mathrm{L}\right)$ entre o resultado da Etapa 2 e [23] observa-se a menor taxa de desgaste em $V_{c}$ mais baixas em ambos os resultados, ver Tabela 6 . 
A taxa de desgaste para a $V_{c}=190 \mathrm{~m} / \mathrm{min}$ é quase três vezes maior que a taxa de desgaste para $\mathrm{V}_{\mathrm{c}}=150$ $\mathrm{m} / \mathrm{min}$, corroborando que a menor taxa de desgaste da ferramenta também não aconteceu na velocidade ótima de corte encontrada na Etapa 1.

Tabela 6: Comparação das taxas de desgaste

\begin{tabular}{l|l|l|l}
\hline \multicolumn{2}{l|}{ ETAPA 2 } & \multicolumn{2}{l}{ MITSUBISHI MATERIALS [23] } \\
\hline $\mathbf{V}_{\mathbf{c}}[\mathrm{m} / \mathbf{m i n}]$ & VB $_{\mathrm{B}} / \mathrm{L}[\mathrm{mm} / \mathbf{k m}]$ & $\mathrm{V}_{\mathrm{c}}[\mathrm{m} / \mathbf{m i n}]$ & VB $_{\mathrm{B}} / \mathrm{L}[\mathrm{mm} / \mathbf{k m}]$ \\
\hline $\mathbf{7 0}$ & 0,015 & $\mathbf{1 5 0}$ & 0,027 \\
\hline $\mathbf{1 1 0}$ & 0,018 & $\mathbf{1 7 0}$ & 0,045 \\
\hline $\mathbf{2 3 0}$ & 0,041 & $\mathbf{1 9 0}$ & 0,077 \\
\hline $\mathbf{3 5 0}$ & 4,967 & $\mathbf{2 0 0}$ & 0,254 \\
\hline
\end{tabular}

Contudo, é possível elaborar algumas considerações metalúrgicas acerca do maior desgaste encontrado para a $\mathrm{V}_{\mathrm{c}}$ próxima da $\mathrm{V}_{\text {copt }}$. $\mathrm{O}$ resultado da análise química por espectrometria ótica (Tabela 1) indicou baixos teores de $\mathrm{S}$ e $\mathrm{Al}$ no aço dos corpos de prova, consequentemente baixa probabilidade de formação de precipitados de $\mathrm{MnS}$ e óxidos de alumínio complexos. Conforme exposto em [9], em temperaturas elevadas (correspondendo com a faixa de temperatura do vale de ductilidade do aço) e alta taxa de deformação (característica peculiar da operação de corte no torneamento) estão associados à precipitação de $\mathrm{MnS}$ nos contornos de grão causando fragilização do aço, ou seja, contribuiria para o vale de ductilidade. Além disso, conforme [10] a formação de óxidos de alumínio na faixa de temperatura de $600{ }^{\circ} \mathrm{C}$ a $800{ }^{\circ} \mathrm{C}$ também poderia contribuir para $\mathrm{o}$ vale de ductilidade. Assim, é admissível que para as $\mathrm{V}_{\mathrm{c}}$ maiores e, portanto temperaturas mais elevadas o vale de ductilidade pode ter apresentado uma curvatura menos acentuada, o que deixaria o aço mais dúctil nessa faixa de temperatura, aumentado o desgaste da ferramenta.

\section{CONCLUSÕES}

Diante dos resultados apresentados e da discussão desenvolvida ao longo deste trabalho, pode-se concluir que não foi possível validar a existência de uma temperatura ótima de corte no torneamento a seco do aço ABNT 1045 utilizando insertos de metal duro por meio da menor força de corte estabilizada, pois foram encontradas diferentes temperaturas correspondentes ao início da estabilização das forças.

Do mesmo modo, não foi possível validar a existência de uma temperatura ótima de corte por meio da melhor qualidade da superfície usinada medindo-se a rugosidade por meio do parâmetro Ra. As curvas dos valores de rugosidade Ra tiveram variações pequenas e aleatórias em função do aumento da temperatura e velocidade de corte.

A velocidade ótima de corte encontrada pelo método menor força de corte estabilizada para o avanço $\mathrm{f}$ $=0,3 \mathrm{~mm} / \mathrm{rev}\left(\mathrm{V}_{\text {copt }}=190 \mathrm{~m} / \mathrm{min}\right)$ foi diferente do resultado de menor desgaste $\left(\mathrm{V}_{\mathrm{c}}=70 \mathrm{~m} / \mathrm{min}\right)$ obtido nos três modos de medição de desgaste (perda de massa, desgaste de flanco e de entalhe).

As forças de usinagem apresentaram os maiores valores para a $V_{c}=70 \mathrm{~m} / \mathrm{min}$ embora esta $V_{c}$ tenha apresentado o menor desgaste em $\mathrm{f}=0,3 \mathrm{~mm} / \mathrm{rev}$. Com isso, constatou que o menor desgaste ou menor taxa de desgaste não ocorreu na mínima força de corte, contrariando nesse ponto a Lei de Makarow.

\section{AGRADECIMENTOS}

Os autores desta pesquisa agradecem a assistência do Departamento de Engenharia Mecânica da Universidade Federal de Minas Gerais (UFMG) pela disponibilização do Laboratório de Usinagem e Automação. Agradecem, também, ao Laboratório de Ensaios e Análises em Materiais (LAMAT) do Serviço Nacional de Aprendizagem Industrial (SENAI) em Itaúna, Minas Gerais pela análise química por espectrometria de emissão ótica dos corpos de prova. À Capes e ao CNPq pelo financiamento desta pesquisa. 


\section{BIBLIOGRAFIA}

[1] TRENT, E.M., WRIGHT, P.K., Metal Cutting, 4. ed., United States of America, Butterworth-Heinemann, 2000.

[2] DAVOODI, B., TAZEHKANDI, A.H., Experimental investigation and optimization of cutting parameters in dry and wet machining of aluminum alloy 5083 in order to remove cutting fluid, Journal of Cleaner Production, [S. l.], v. 68, pp. 234-242, 2014.

[3] YUSUP, N., ZAIN, A.M., HASHIM, S.Z.M. Evolutionary techniques in optimizing machining parameters: Review and recent applications (2007-2011), Expert Systems with Applications, [S. l.], v. 39, p. 99099927, 2012.

[4] SHENG, J., A modeling method for turning parameters coupling based on minimum cutting tool wear, Int J Adv Manuf Technol, London, v. 76, pp. 705-712, 2014.

[5] SHENG, J., CHIU, Y., LIN, B., Determination of a coupling equation for milling parameters based on optimal cutting temperature, Int J Adv Manuf Technol, London, 2017.

[6] ANUKHIN, I.V., et al., Thermal Imaging in Selecting the Cutting Conditions for HighTemperature Intermetallic Alloys, Russian Engineering Research, St. Petersburg, v. 35, n. 7, pp. 544-548, 2015.

[7] ASTAKHOV, V.P., Tribology of Metal Cutting, 1. ed., Great Britain, Elsevier, 2006.

[8] HAO, Z.P., et al., Wear characteristics and wear control method of PVD-coated carbide tool in turning Inconel 718, Int J Adv Manuf Technol, London, v. 78, pp. 1329-1336, 2015.

[9] ASM HANDBOOK FRACTOGRAPHY, Volume 12 - Fractography, 9.ed., USA, ASM Internation, 1987.

[10] CAGALA, M., et al., Mechanical properties of two manganese steels, Archives of Foundry Engineering, v. 12, pp. 9-12, Czech Republic, 2012.

[11] MITSUBISHI MATERIALS, Catálogo Geral C008Z - Insertos de Torneamento Classes de Insertos, [S. l.: s. n.], pp. 168, 2018.

[12] HEIGEL, J.C., WHITENTON, E.P., The effects of emissivity and camera point spread function on the temperature measurement of segmented chip formation using infrared thermography, International Manufacturing Science and Engineering Conference, Pennsylvania, USA, 2010.

[13] OLIVEIRA, J.A, Estudo do processo de rosqueamento por conformação na liga de alumínio 7078-T6, Dissertação (Mestrado em Engenharia Mecânica) - Pós Graduação Engenharia Mecânica, UFSJ, São João Del Rei, 2016.

[14] INTERNATIONAL STANDARD, ISO 3685: Tool life testing with single - point turning tools. 2.ed., Switzerland: ISO, 1993.

[15] RAYTEK, Operating Instructions - High-Performance Infrared Thermometer: Marathon - MM Series. Rev. C1, p. 88, 2007.

[16] KISTLER, Instruction Manual, Dynamometer Type 9272, Quartz 4 - Component, 2008.

[17] KISTLER, Instruction Manual, Charge Amplifier for Manufacturing Applications Type 5073A, 2012.

[18] PANZERA, T.H., et al., Development of a three-component dynamometer to measure turning force, Int J Adv Manuf Technol, London, pp. 913-922, 2012.

[19] NOORDIN, M.Y., et al., Application of response surface methodology in describing the performance of coated carbide tools when turning AISI 1045 steel, Journal of Materials Processing Technology, Malaysia, pp. 46-58, 2003.

[20] FERRARESI, D., Fundamento da Usinagem dos Metais, 1. ed., São Paulo, Edgard Blucher, 1970, v. 1.

[21] SHAW, M.C., Metal Cutting Principles. 2. ed. New York: Oxford University Press, 2005.

[22] MACHADO, A.R., et al., Teoria da Usinagem dos Materiais, 1. ed. São Paulo, Blucher, 2009.

[23] MITSUBISHI MATERIALS, Efeitos das Condições de Corte para Torneamento, Disponível em: http://www.mitsubishicarbide.com/application/files/7215/1859/7499/tec_turning_effects_pt-br.pdf , Acesso em 11 de jan. 2019.

\section{ORCID}

Verner Petersen Pereira Marcelo Araújo Câmara
https://orcid.org/0000-0001-8694-048X

https://orcid.org/0000-0003-0617-9163 